\title{
Gradient-index electron optics in graphene pn junctions
}

\author{
Emmanuel Paredes-Rocha, ${ }^{1, \text { f } Y o n a t a n}$ Betancur-Ocampo,, , † Nikodem Szpak, ${ }^{2}$, 周and Thomas Stegmann ${ }^{1,8}$ \\ ${ }^{1}$ Instituto de Ciencias Físicas, Universidad Nacional Autónoma de México, Cuernavaca, Mexico \\ ${ }^{2}$ Fakultät für Physik, Universität Duisburg-Essen, Duisburg, Germany
}

\begin{abstract}
We investigate the electron transport in smooth graphene pn junctions, generated by gradually varying electrostatic potentials. The numerically calculated coherent current flow patterns can be understood largely in terms of semi-classical trajectories, equivalent to the ones obtained for light beams in a medium with a gradually changing refractive index. In smooth junctions, energetically forbidden regions emerge, which increase reflections and can generate pronounced interference patterns, for example, whispering gallery modes. The investigated devices do not only demonstrate the feasibility of the gradient-index electron optics in graphene pn junctions, such as Luneburg and Maxwell lenses, but may have also technological applications, for example, as electron beam splitters, focusers and waveguides. The semi-classical trajectories offer an efficient tool to estimate the current flow paths in such nano-electronic devices.
\end{abstract}

\section{INTRODUCTION}

The ballistic, beam-like propagation of electrons in graphene enables the observation of optical-like phenomena in this material. This electron optics has recently come into focus of research with several theoretical and experimental contributions [1-16]. For example, it has been shown that an electron beam, which hits the interface of a graphene pn junction, behaves like a light beam at the interface of two materials with different refractive indices. Hence, the reflection and refraction of the electrons follows a generalized version of Snell's law, where the refractive indices are determined by the electrostatic potential in the $\mathrm{p}$ and $\mathrm{n}$ region of the junction [1, 6, 9, 10, 17 19]. Due to the special dispersion relation of graphene, negative reflection can be observed, a property that has been seen before only in the light propagation in metamaterials 20 22. Moreover, Klein tunneling - the absence of backscattering at normal incidence - is observed, which can be attributed to the pseudo-spin conservation of the electrons in graphene. It has also been shown that an electron beam in graphene can be deflected by means of elastic deformations that induce a strong pseudo-magnetic field 2328 .

The possibility to manipulate electron beams in graphene by means of pn junctions or elastic deformations has lead to various proposals for nano-electronic devices, such as Veselago lenses [4, 6, 29, 35, electron fiber optics 3, 36, interferometers 37, 38, valley beam splitters [7, 26, 39 50, collimators [51, 52, switches [53, reflectors [54, 55], transistors 22, 56, 57, and Dirac fermions microscopes 58 . Electron optics has been extended recently from graphene to other materials, such as phosphorene where negative reflection has been pre-

\footnotetext{
* eparedes@icf.unam.mx

† ybetancur@icf.unam.mx

$\ddagger$ nikodem.szpak@uni-due.de

$\S$ stegmann@icf.unam.mx
}

dicted 59, 60, non-coplanar refraction and Veselago lenses in Weyl semi-metals 61 64, anomalous caustics in borophene pn junctions [65], and super-diverging lenses in Dirac materials [30].

Most of the work on electron optics in graphene pn junctions involves interfaces where the electrostatic potential (and hence the refractive index) changes abruptly [8, 29, 35, 66, 68. Recent experiments have demonstrated that such abrupt junctions can indeed be realized [13. Pn junctions with a smoothly changing electrostatic potential are often regarded as disadvantageous, because they induce an energetically forbidden region and hence, reduce the transmission. Nevertheless, one can also take advantage of the reduced transmission to construct quantum dots based on smooth circular pn junctions. These junctions show interesting physical properties like Mie scattering [29] and whispering gallery modes [69]. They have been realized recently in experiments [4, 70].

A smoothly changing electrostatic potential can be understood as a smoothly changing refractive index that establishes gradient-index optics. In this paper, we investigate to which extent graphene pn junctions show gradient-index optics phenomena. We will study straight pn junctions as well as circular junctions which have received little attention so far. On the one hand side, we will calculate numerically the current flow in these systems, applying the non-equilibrium Green's function method to the tight-binding model. On the other hand side, using the geometric optics approximation, we will determine the semi-classical trajectories of the electron beams. Comparing both approaches we will show that they agree in a wide regime of parameters. Nevertheless, discrepancies emerge which can be explained by the interference of electron waves. These wave effects increase for smooth circular junctions as they can partially confine the electron beam. 


\section{SYSTEM \& METHODS}

\section{Graphene pn junctions}

We model the electronic structure of graphene by the tight-binding Hamiltonian

$$
H=-t \sum_{i, j}\left|i^{A}\right\rangle\left\langle j^{B}\right|+\text { H.c. }
$$

which describes well the electron transport at low energies. The $\left|i^{A / B}\right\rangle$ indicate the atomic states localized on the carbon atoms at positions $\boldsymbol{r}_{i}$ on the sublattices A and B, respectively. The sum runs over nearest neighboring atoms, which are separated by a distance of $a=0.142 \mathrm{~nm}$ and coupled with the energy $t=2.8 \mathrm{eV}$. A plane-wave ansatz leads at low energies to the continuous Dirac Hamiltonian

$$
H_{\mathrm{Di}}^{ \pm}(\boldsymbol{k})=\hbar v_{F} \boldsymbol{\sigma}_{ \pm} \cdot \boldsymbol{k}
$$

at the two Dirac points $\boldsymbol{K}_{ \pm}=(0, \pm 4 \pi /(3 \sqrt{3} a))$ at the edges of the Brillouin zone. The wavevector $\boldsymbol{k}$ is measured with respect to these Dirac points. We define $\hbar v_{F}=3 a t / 2$ and $\boldsymbol{\sigma}_{-}=\boldsymbol{\sigma}_{+}^{*}=\left(\sigma_{1}, \sigma_{2}\right)$. The valley degree of freedom of the electrons in graphene, which may be used for a new kind of electronics called valleytronics [41, 43 48, will not be relevant in the present work, because the considered pn junctions do not affect it. The Dirac Hamiltonian leads to the well-known conical energy bands of graphene

$$
E(\boldsymbol{k})=s \hbar v_{F}|\boldsymbol{k}|
$$

where $s=\operatorname{sign}(E)= \pm 1$ is the band index.

A graphene pn junction is constituted by regions of different doping, see Figure 1. Such regions can be generated by metallic gates that induce an electrostatic potential, $V(\boldsymbol{r})$, in the continuous space representation or $V=\sum_{i} V_{i}|i\rangle\langle i|$ in the tight-binding approach. This potential shifts the energy bands of graphene and hence, generates a junction. A pn junction is generated if the electrons go from one band to another through interband tunneling, while in a pp' or nn' junction intraband tunneling takes place within the valence or conduction band, respectively. In the following, we will concentrate on straight pn junctions (Figure 1 (a)), generated by the electrostatic potential

$$
V_{\operatorname{lin}}(\boldsymbol{r})=\left\{\begin{array}{lll}
0 & \text { if } & x \leq-w / 2 \\
\left(\frac{x}{w}+\frac{1}{2}\right) V & \text { if } & |x|<w / 2 \\
V & \text { if } & x \geq w / 2
\end{array}\right.
$$

and circular junction (Figure 1 (b)) with the potential

$$
V_{\text {cir }}(\boldsymbol{r})=\frac{V}{1+\left(r / r_{0}\right)^{\alpha}}
$$

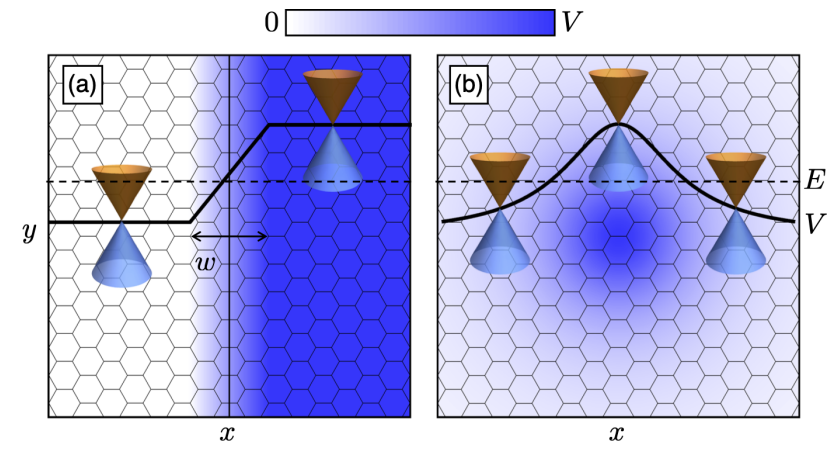

Figure 1. Straight (a) and circular (b) graphene pn junctions. The bluish color shading shows the electrostatic potential within the graphene ribbon. Both junctions have a smooth interface that separates the two regions of different doping. The profile of the electrostatic potential is indicated by the solid black lines, which shifts locally the conical energy bands of graphene. This causes that the electrons at energy $E$ (black dashed lines) go from the conduction band (orange cone) to the valence band (light blue cone).

The smoothnes: ${ }^{1}$ of the pn junctions is controlled by the parameters $w$ and $\alpha$. Note that circular pn junctions can be also understood as pnp junctions because the electrostatic potential is vanishing for $r \rightarrow \infty$.

\section{Semi-classical trajectories}

Within semi-classical theory, the propagation of the electron wave functions is approximated by the propagation of point particles. In order to apply this approximation to the quantum system described by the Dirac Hamiltonian Equation (2), we use the eikonal approximation and the methods developed in 26] to obtain relativistic trajectories (geodesics) for massless particles coupled to the electric potential $V(\boldsymbol{r})$

$$
\frac{d \boldsymbol{p}}{d t} \equiv \frac{d}{d t}\left(\frac{(E-V(\boldsymbol{r}))}{v_{F}^{2}} \frac{d \boldsymbol{r}}{d t}\right)=-\nabla V(\boldsymbol{r}),
$$

where the momentum vector $\boldsymbol{p}(t)$ along the trajectory $\boldsymbol{r}(t)$ satisfies the dispersion relation $(E-V(\boldsymbol{r}))^{2}=v_{F}^{2} \boldsymbol{p}^{2}$. This approach is discussed in detail in our previous works and applied successfully to understand the current flow paths in elastically deformed graphene [26, 39] .

In order to get additional insight, the dynamics can be reformulated by means of the classical pseudo-relativistic Hamiltonian

$$
H_{\mathrm{sc}}=s(\boldsymbol{r}) v_{F}|\boldsymbol{p}|+V(\boldsymbol{r}),
$$

where $s(\boldsymbol{r})=\operatorname{sign}(E-V(\boldsymbol{r}))$ is the band-index and $|\boldsymbol{p}|=$ $\sqrt{p_{x}^{2}+p_{y}^{2}}=\sqrt{p_{r}^{2}+p_{\theta}^{2} / r^{2}}$ is the momentum in Cartesian

\footnotetext{
${ }^{1}$ Note that mathematical smoothness is not relevant here.
} 
and polar coordinates, respectively. The trajectories of the ballistic electrons described by this Hamiltonian can be related to optical rays in an artificial medium with the refractive index

$$
n(\boldsymbol{r}) \equiv \frac{E-V(\boldsymbol{r})}{v_{F}} .
$$

Taking into account that the electrostatic potential $V(\boldsymbol{r})$ can change smoothly (on the length scale defined by the Fermi wavelength of the electrons), we obtain in this way a gradient-index medium.

The equations of motion in a straight junction are given by

$$
\begin{aligned}
& \frac{d x}{d t}=\partial_{p_{x}} H=\frac{s(x) v_{F} p_{x}}{\sqrt{p_{x}^{2}+p_{y}^{2}}}=\frac{v_{F} p_{x}}{n(x)} \\
& \frac{d y}{d t}=\partial_{p_{y}} H=\frac{s(x) v_{F} p_{y}}{\sqrt{p_{x}^{2}+p_{y}^{2}}}=\frac{v_{F} p_{y}}{n(x)} .
\end{aligned}
$$

Eliminating the time dependency by dividing both expressions, we obtain for the semi-classical trajectories

$$
y(x)=y_{0}+p_{y} \int_{x_{0}}^{x} \frac{s\left(x^{\prime}\right) d x^{\prime}}{\sqrt{n^{2}\left(x^{\prime}\right)-p_{y}^{2}}},
$$

where $\boldsymbol{r}_{0}=\left(x_{0}, y_{0}\right)$ is the initial position of the electron. A similar analysis for the electron trajectories in circular junctions leads to

$$
\theta=\theta_{0}+l \int_{r_{0}}^{r} \frac{s\left(r^{\prime}\right) d r^{\prime}}{r^{\prime} \sqrt{r^{\prime 2} n^{2}\left(r^{\prime}\right)-l^{2}}}
$$

where $l \equiv p_{\theta}$ is the angular momentum. Note that the momentum component $p_{y}$ is conserved in straight junctions due to the translational symmetry along the $y$-axis (see Figure 1 (a)), while the angular momentum $\equiv p_{\theta}$ is conserved in circular junctions due to the rotational symmetry (see Figure 1(b)).

These electron trajectories are identical to the ones obtained for light beams in gradient-index optics [71, apart from an important difference: The refractive index $n(\boldsymbol{r})$ depends on the electron energy and changes its sign when the electrons go from the conduction (n region) to the valence band ( $\mathrm{p}$ region), see Figure 2. It is all negative for pp' and positive for nn' junctions. Moreover, the square root in the denominator of Eqs. (10) and (11) can become imaginary in certain regions of the system, which are defined by the inequalities $|n(x)| \leq\left|p_{y}\right|$ and $|n(r)| \leq|l| / r$ for straight and circular junctions, respectively. These forbidden regions are indicated in Figure 3 by those ranges where the refractive index (bluishreddish curve) lies in the gray shaded regions. While classically those regions cannot be penetrated, quantummechanically the electrons can tunnel through the forbidden regions.

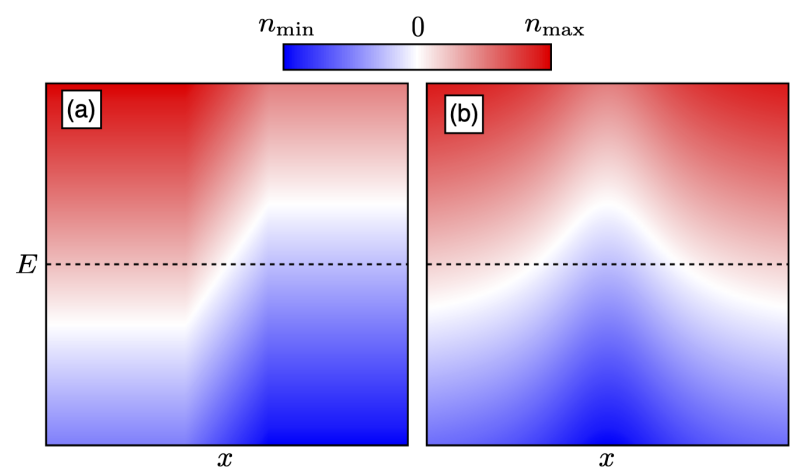

Figure 2. Refractive index $n$ in a straight (a) and circular (b) graphene pn junction as a function of the position $x$ (compare Figure 1) and the electron energy $E$. Reddish colors indicate $n>0$, while bluish colors represent $n<0$. Note that for certain electron energies (dashed horizontal line) $n$ changes its sign in the narrow white region.
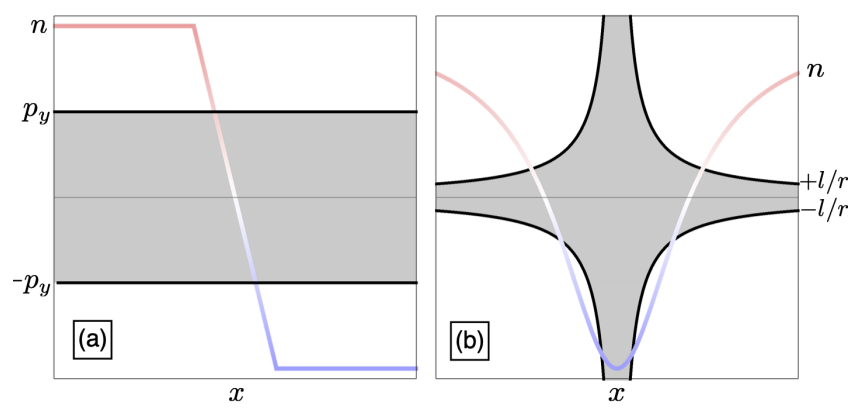

Figure 3. The reddish-bluish curve gives the refractive index $n$ at constant electron energy, see the dashed horizontal curves in Figure 2. The black curves represent the momentum component $p_{y}$ and $l$ in straight (a) and circular (b) junctions, respectively. When $n$ is in the gray shaded regions, the root in Eqs. 10 and 11 is imaginary and the electrons have to tunnel through a forbidden region.

As tunneling is largely suppressed in smooth junctions, the boundary of the forbidden region defines the reflection zone for the beam. Figure 3 explains that the transmission decreases if the incidence of the electrons becomes more oblique, because $p_{y}$ (or $l$ ) increases and, thereby, the size of the forbidden region increases, too. In the same way, the transmission is perfect for normal incidence, because $p_{y}=0$ (or $l=0$ ) and the forbidden region disappears, which matches with the pseudo-spin conservation. The semi-classical trajectories obtained from the geometrical optics are equivalent to the relativistic geodesics in the classical region $(n>0)$, but offer additional techniques to deal with non-classical phenomena like tunneling though classically forbidden regions. Note also that the refractive index in Figure 2 (b) is astonishingly similar to a measurement of the local resistance in [4]. 


\section{The non-equilibrium Green's function method for the current flow}

The current flow in the graphene pn junction is calculated by means of the non-equilibrium Green's function (NEGF) method. This quantum method is based on the tight-binding Hamiltonian, Equation (1). It does not rely on the approximations made in the previous section to obtain the semi-classical trajectories and thus allows us to verify their validity. As the NEGF method is discussed in detail in various textbooks, see e.g. [72, 73, we summarize here only briefly the essential formulas.

The Green's function of the system is given by

$$
G(E)=(E-H-V-\Sigma)^{-1},
$$

where $E$ is the energy of the injected electrons, $H$ is the tight-binding Hamiltonian, Equation (1), and $V$ the electrostatic potential. In order to suppress boundary effects and mimic an infinite system, we place a constant complex potential $\Sigma=-\mathrm{i} \sum_{i \in \text { edges }}|i\rangle\langle i|$ at the edges of the system, which should absorb the electrons.

The electrons are injected at the left edge of the system as plane waves propagating towards the interface of the pn junction. Their momentum is determined by the electron energy, Equation (3), and the angle of injection $\theta=\arctan k_{y} / k_{x}$. This injection is represented by the inscattering function

$$
\Sigma^{\text {in }}=\sum_{i, j \in \text { edge }} A\left(\boldsymbol{r}_{i}\right) A\left(\boldsymbol{r}_{j}\right) \psi_{j}{ }^{*}(\boldsymbol{k}) \psi_{i}(\boldsymbol{k})|i\rangle\langle j|,
$$

where the sum runs over all carbon atoms at the left system edge, see Figure 1 The $\psi_{i}(\boldsymbol{k})$ are the eigenstates of the Dirac Hamiltonian, Equation (2),

$$
\psi_{i}(\boldsymbol{k})= \begin{cases}c^{-} e^{\mathrm{i}\left(\boldsymbol{k}+\boldsymbol{K}^{-}\right) \boldsymbol{r}_{i}}+c^{+} e^{\mathrm{i}\left(\boldsymbol{k}+\boldsymbol{K}^{+}\right) \boldsymbol{r}_{i}} & i \in A, \\ s c^{-} e^{\mathrm{i}\left(\boldsymbol{k}+\boldsymbol{K}^{-}\right) \boldsymbol{r}_{i}+\mathrm{i} \phi}-s c^{+} e^{\mathrm{i}\left(\boldsymbol{k}+\boldsymbol{K}^{+}\right) \boldsymbol{r}_{i}-i \phi} & i \in B,\end{cases}
$$

where $\phi=\arg \left(\mathrm{i} k_{x}+k_{y}\right)$. The parameters $c^{ \pm}$control the occupation of the two $\boldsymbol{K}^{ \pm}$valleys. We consider the case in which both valleys are fully mixed, i.e. $c_{ \pm}= \pm 1 / 2$. The function

$$
A(\boldsymbol{r})=e^{-\left(y-y_{0}\right)^{2} / d_{0}^{2}}
$$

gives the injected current beam a Gaussian profile. The parameters $y_{0}$ and $d_{0}$ control the position and width of the beam.

Finally, the current flowing between the atoms at positions $\boldsymbol{r}_{i}$ and $\boldsymbol{r}_{j}$ is calculated by

$$
I_{i j}=\operatorname{Im}\left(t G \Sigma^{\operatorname{in}} G^{\dagger}\right)_{i j} .
$$

Good agreement between the quantum current flow and the semi-classical trajectories of geometric optics can be expected only in the specific parameter regime, where the Fermi wavelength of the electrons $\lambda_{F}$ is much larger than the interatomic distance $a$ but smaller than the system size $\left(L_{x}, L_{y}\right)$. Moreover, smooth changes of the electrostatic potential, as sketched in Figure 1. can be resolved only if the Fermi wavelength is shorter than the spatial variations of the potential $\Delta_{x / y} V$. These conditions can be summarized in the inequality

$$
a \ll \lambda_{F}=\frac{2 \pi}{|\boldsymbol{k}|}<\Delta_{x / y} V \ll L_{x / y} .
$$

However, when the electrostatic potenial changes smoothly the effective electron energy $E-V$, and hence the Fermi wavelength, will change which may lead to a local violation of the inequality.

\section{RESULTS}

\section{Straight graphene pn junctions}

We begin our discussion by analyzing the current flow in a homogeneous graphene nanoribbon with a size of about $150 \times 150 \mathrm{~nm}$ or larger ${ }^{2}$ In Figure 4 (a), electrons are injected according to Equation $(13)$ at the left ribbon edge with energy $E=0.15 t \approx 420 \mathrm{meV}$ (corresponding to the Fermi wavelength $\lambda_{F} \approx 9 \mathrm{~nm}$ ) and a momentum vector parallel to the horizontal $x$-axis. A beam-like propagation of the electrons with some divergence due to diffraction is observed, which will enable us to compare the numerically calculated current flow patterns with the semi-classical trajectories.

In Figure 4 (b) a straight pn junction is introduced by an electrostatic potential which changes abruptly from zero to the constant value $V=2 E$ at the dashed line. We observe negative refraction at the interface of the pn junction which re-focuses the electron beam. Moreover, Klein tunneling, i.e. the absence of back-scattering at normal incidence, occurs. When the junction is tilted ${ }^{3}$ the electrons hit the interface no longer orthogonally and the incident electron beam is split into a reflected and refracted beam. The solid black, blue and green lines in Figure 4 are the predicted semi-classical trajectories (Equation (10) for the incident, transmitted and reflected electron beams, respectively. They follow a generalized Snell's law,

$$
\theta_{\mathrm{re}}=-\theta_{\mathrm{in}} \quad \text { and } \quad \frac{\sin \left(\theta_{\mathrm{in}}\right)}{\sin \left(\theta_{\mathrm{tr}}\right)}=\frac{n_{\mathrm{tr}}}{n_{\mathrm{in}}}=\frac{E-V}{E},
$$

\footnotetext{
2 The accuracy of the used approximations increases with the system size.

3 Note that due to the isotropy of graphene's electronic structure at low energies, tilting the junction is equivalent to injecting the electrons under a different angle.
} 

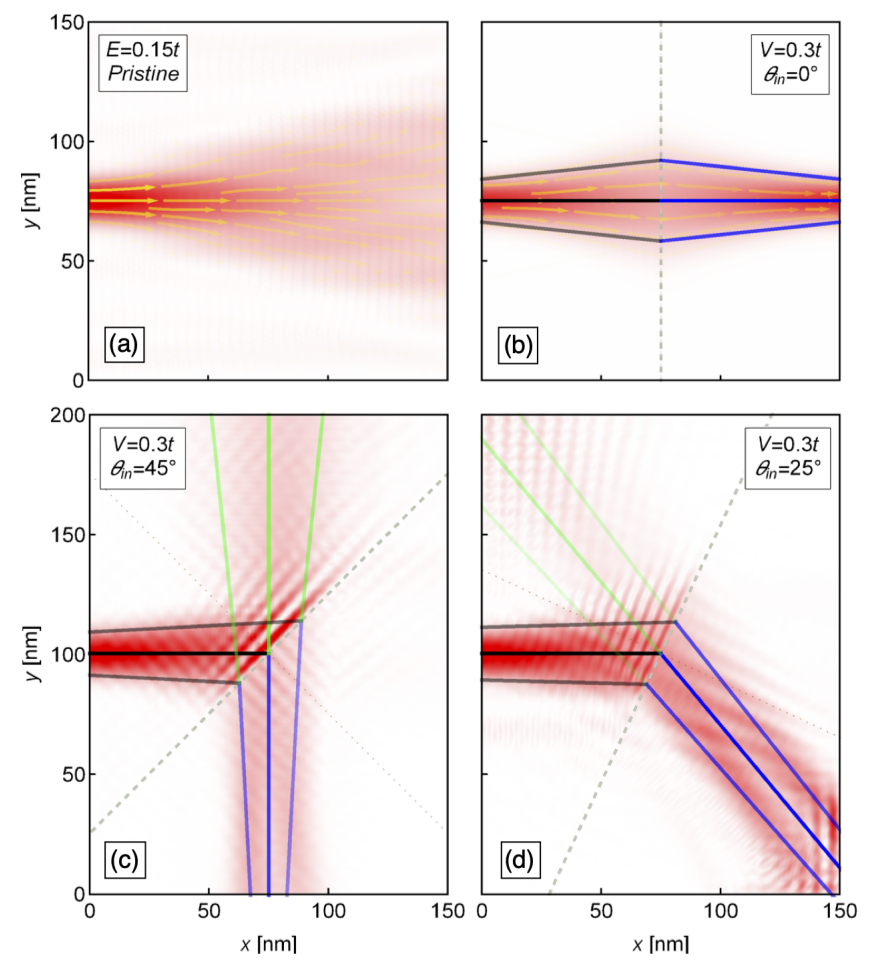

Figure 4. Current flow in a graphene nanoribbon in the absence (a) and in the presence (b,c,d) of an electrostatic potential which changes abruptly at the dashed line and hence, generates a pn junction. The current density is indicated by the red color shading, the current vector field by yellow arrows. The diverging electron beam in (a) is re-focused by the pn junction in (b). The semi-classical trajectories from Equation (10), see the solid black, blue and green lines, agree with the current flow patterns calculated by means of the NEGF method. At the interface of the pn junction, the electron beam is split into a reflected and transmitted electron beam, in agreement with the generalized Snell's law, Equation (18).

where $\theta_{\mathrm{in} / \mathrm{tr} / \mathrm{re}}$ are the angles of incidence, transmission and refraction, while $n_{\text {in/tr }}$ as defined in Equation (8) take the role of the refractive indices in the $\mathrm{n}$ and $\mathrm{p}$ region, respectively. In general, these trajectories agree very well with the numerical quantum calculations, see Figure 4 (b,c,d). Electron optics in such straight pn junctions has been studied largely before [1, 2, 6] 11, 1416, 18, 19, 53, 56, 58, 66, 74, 75. Here, we have confirmed the generalized Snell's law by means of numerical quantum calculations of the current flow. Additionally, we observe in Figure 4 (c,d) a ripple pattern close to the interface of the pn junction, magnified in Figure 5 (a). In Figure 5 (b) we show the superposition of the incoming and reflected electron wave, $\left|e^{\mathrm{i} \boldsymbol{k}_{\mathrm{in}} \cdot \boldsymbol{r}}+e^{\mathrm{i} \boldsymbol{k}_{\mathrm{re}} \cdot \boldsymbol{r}}\right|^{2}$. The agreement of both figures confirms that the ripples are an interference effect of the electron wave functions, which goes beyond semi-classical trajectories. Similar ripple patterns can also be observed close to the edges of the graphene nanoribbon. They can be explained in the same
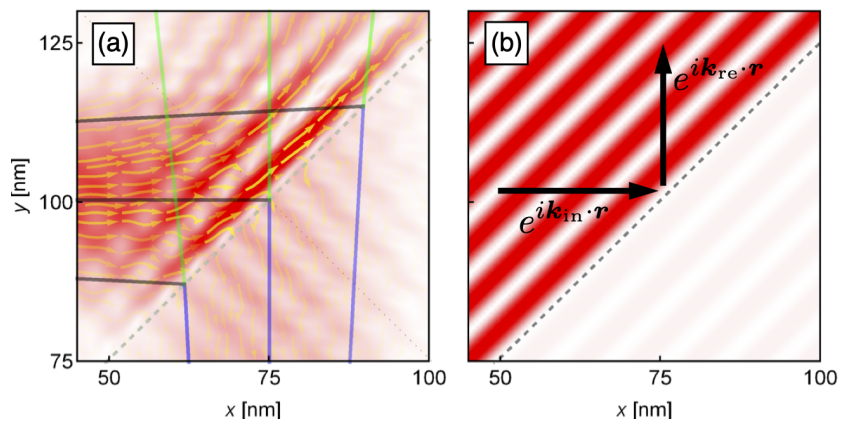

Figure 5. Current flow close to the interface of the graphene pn junction. (a) Zoom at the interface region of Figure 4 (c). (b) Probability density generated by the superposition of the incoming and reflected electron beam, $\left|e^{\mathrm{i} \boldsymbol{k}_{\mathrm{in}} \cdot \boldsymbol{r}}+e^{\mathrm{i} \boldsymbol{k}_{\mathrm{re}} \cdot \boldsymbol{r}}\right|^{2}$. The good agreement of (a) and (b) shows that the interference between the two electron beams generates the ripple pattern.

way by the reflections at the system boundary. The absorption of the electrons at the edges by the complex potential is not perfect and hence generates a small reflected part which interferes with the incoming beam.

We continue our discussion with smooth graphene pn junctions, where the electrostatic potential changes linearly over a width of $w=350 a \approx 50 \mathrm{~nm} \approx 5 \lambda_{F}$, see Figure 1 (a). As shown in Figure 6, the semi-classical trajectories agree well with the current density obtained by means of the NEGF method. The generalized Snell's law remains valid for such smooth junctions. However, in comparison with the case of an abruptly changing potential (see Figure 4), a much larger part of the incident current is reflected. This effect can be observed even for normal incidence, where Klein tunneling takes place due to the diffraction of the electron beam which slightly changes the propagation direction. The effect gets even more pronounced for narrower electron beams for which the diffraction is stronger, compare Figure 6 (a) and (b). Such pn junctions can be used to generate narrow parallel electron beams.

The increasing reflection can be understood by the rise of the forbidden zone (see Figure 3 (a)), which is indicated in Figure 6 by the gray shaded regions. Moreover, an accumulation of the current density can be observed just at the edge of the forbidden zone, which coincides with the point of return of the semi-classical trajectories. Note that we have also sketched trajectories in the forbidden regions by using the substitution $\boldsymbol{k} \rightarrow \mathrm{i} \boldsymbol{k}$, which converts evanescent waves to propagating ones. Although the Fermi wavelength diverges in this region and the geometric approximation might break down the continuation of the trajectories to another classical region agrees again very well with the quantum current.

Until now, we have discussed only the case of pn junctions, where interband tunneling occurs. In the case of nn' and pp' junctions, the electrons remain in the same 

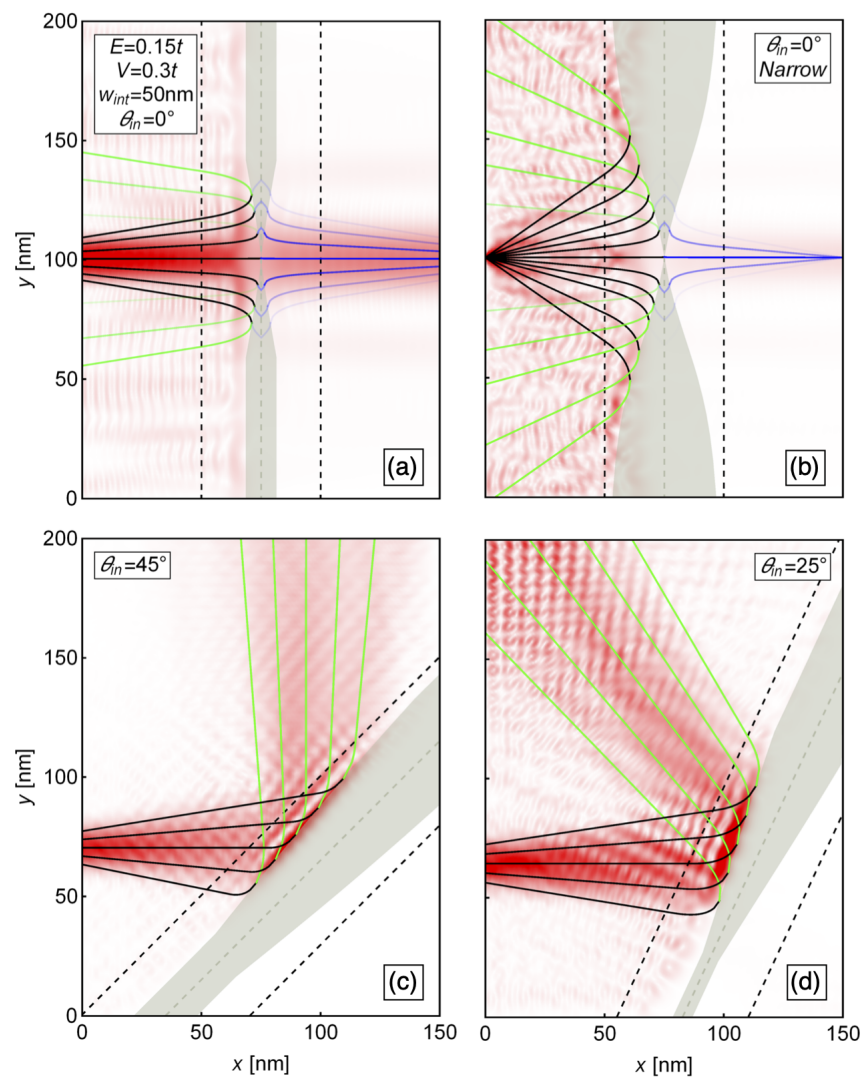

Figure 6. Current flow in a straight graphene pn junction with a smoothly changing profile $(w \sim 50 \mathrm{~nm})$. The width of the junction is indicated by dashed black lines (see Figure 1 (a)). The points where the electrons go from the conduction to the valence band are indicated by a gray dashed line. The semiclassical trajectories (solid lines) agree well with the NEGF current density (red color shading). The forbidden region is indicated by the gray shaded region. At the edge of this region the current density accumulates and the semi-classical trajectories return. Note that the forbidden zone in the case of normal incidence $(a, b)$ appears due to the diffraction of the electron beam, which has been determined on the basis of Figure 4 (a).

band and the current flow patterns change qualitatively. In Figure 7 a narrow (and hence strongly diverging) electron beam is injected at the left ribbon edge. In contrast to the pn junction (see Figure 6 (b)) the current is largely transmitted through the interface of the junction. Moreover, in the nn' junction (Figure 7 (a)) the divergence of the electron beam is enhanced, whereas in the pp' junction (Figure $7(\mathrm{~b})$ ) it is reduced similarly to a converging lens. Note that in nn' junctions a critical angle exists, beyond which total reflection occurs, see the outermost trajectories in Figure 7 (a).
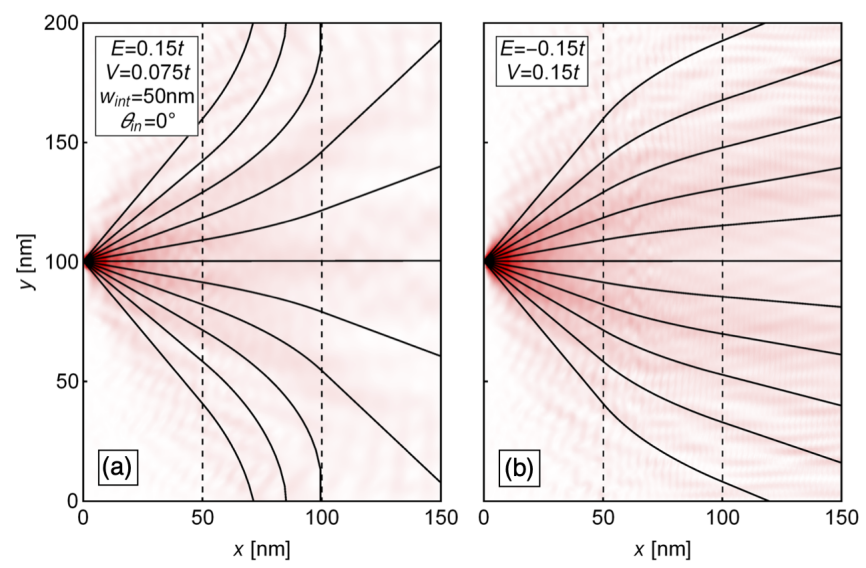

Figure 7. Electron optics in a smooth nn' (a) and pp' (b) junctions. The electrons are injected as a very narrow beam with strong diffraction. The current is transmitted largely through the junction in contrast to the pn junction, where the electrons are transmitted only at normal incidence (compare Figure6(b)).
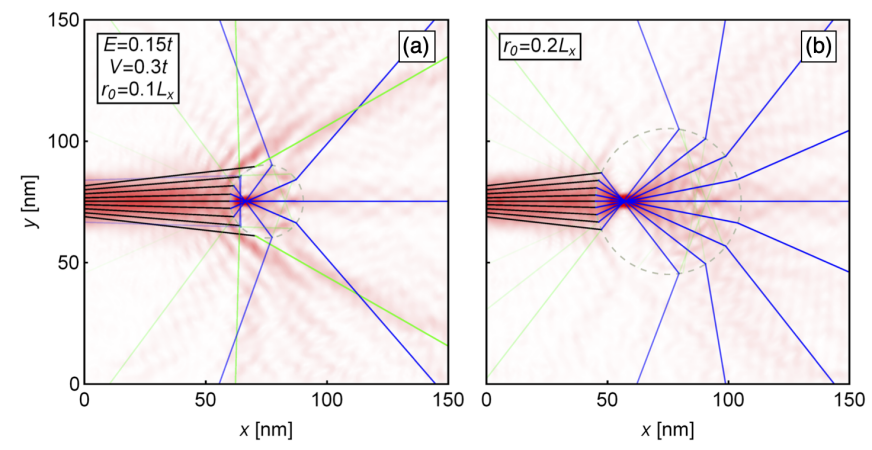

Figure 8. Current flow in a circular graphene pn junction. The electrostatic potential is changing abruptly at the dashed circle from zero to $V=2 \mathrm{E}$. A single focusing point emerges inside the junction, whose interface is denoted by the dashed circle. Outside the junction, the electrons are scattered divergently. The semi-classical trajectories agree well with the current flow inside the junction, but not that well outside due to interference of the incoming and reflected electron waves in a wide region.

\section{Circular graphene pn junctions}

Let us consider now the circular graphene pn junctions. As in the case of straight junctions, we begin with an abruptly changing electrostatic potential, obtained by $\alpha \rightarrow \infty$ in Equation (5). In Figure 8, it can be observed that a part of the current density is deflected around the pn junction while the part that enters the junction is focused onto a single point [5]. The semiclassical trajectories show a caustic inside the junction in agreement with the focusing point observed in the current density. However, outside the junction both approaches are much less in line. This disagreement can 


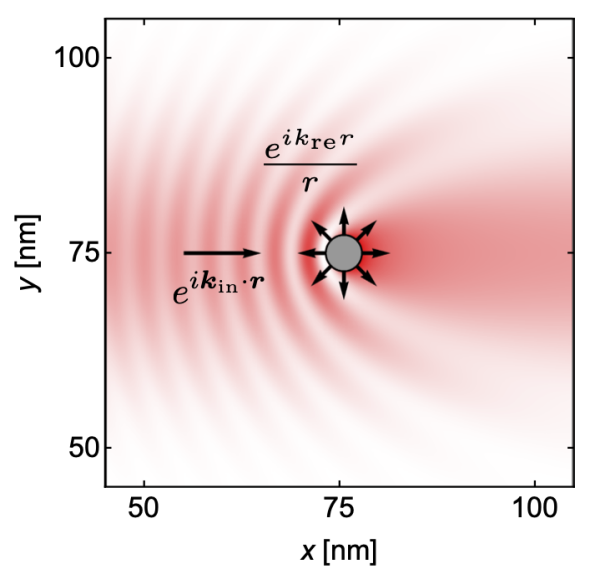

Figure 9. Interference of an incoming plane wave (with Gaussian profile, see Equation (15) and a reflected circular electron beam, $\left|A(\boldsymbol{r}) e^{\mathrm{i} \boldsymbol{k}_{\mathrm{in}} \cdot \boldsymbol{r}}+e^{\mathrm{i} k_{\mathrm{re}} r}\right|^{2}$ shows that the current pattern outside the junction is changed strongly by interference.

be explained by the wave nature of the electrons which leads in straight junctions to a ripple pattern at the interface of the junction due to the interference between the incoming and reflected electron waves. In circular junctions, the reflected electron wave is (approximately) circular and hence interferes with the incident wave in a much larger region. The resulting interference pattern shown in Figure 9 agrees qualitatively with the observed current pattern and demonstrates the limitations of the geometric optics in circular pn junctions. Additionally, we note in Figure 8 that when the radius of the junction is reduced, the incidence of lateral electrons becomes more grazing and a larger part of the electrons is deflected around the junction.

By smoothing the profile of the electrostatic potential $(\alpha=2)$, we obtain a circular junction with a gradually changing refractive index, which is certainly the most appealing and novel device for gradient-index electron optics. Such a device has been realized recently experimentally [4, 70]. Current flow patterns are shown in Figure 10 together with semi-classical trajectories, which agree roughly with the quantum current. The disagreement can be explained by strong interference due to an energetically forbidden region through which the electrons have to tunnel. Therefore, a large fraction of the incident electron beam is reflected, which causes a ripple pattern that is much more pronounced than in abrupt circular junctions, see Figure 8. Similar to smooth straight junctions, see Figure 6 an accumulation of the current density can be observed at the edges the forbidden region, which is indicated in the semi-classical trajectories by a color change from black to green. The size of the forbidden region is minimal for the electrons of normal incidence (see Figure 3) and hence, those electrons enter and leave the junction preferentially. Those elec-
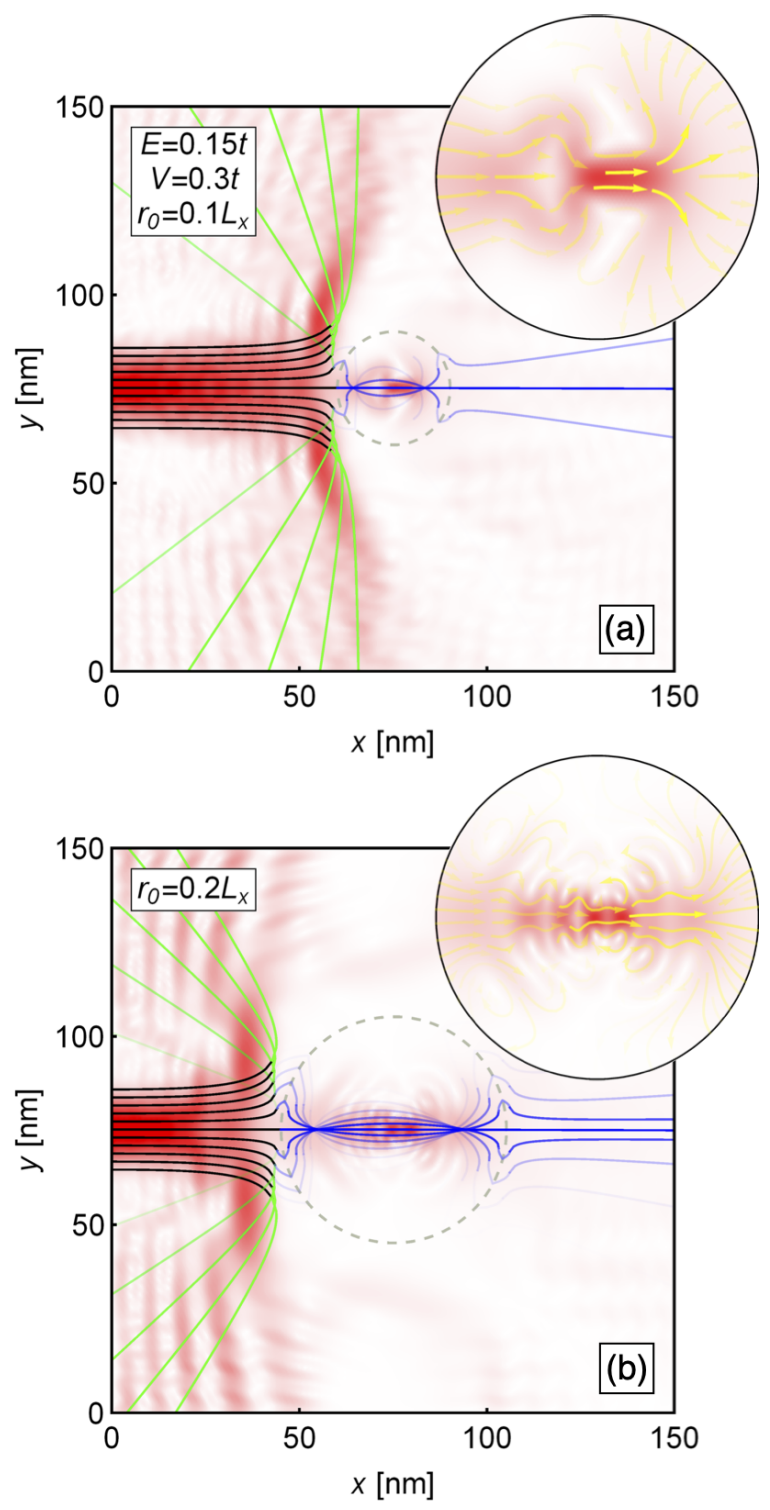

Figure 10. Gradient-index electron optics in smooth circular pn junctions $(\alpha=2)$. The dashed circle indicates the isoline $V(r)=E$. The current flow pattern and the semi-classical trajectories agree roughly. The differences can be explained by the existence of an energetically forbidden region, which enhances reflections outside the junction as well as the confinement inside the junction and hence, leads to pronounced interference patterns. The interface of the forbidden region is indicated by the point where the trajectories change their color from black to green. The inset shows a zoom close to the center of the pn junction.

trons that enter the junction are strongly confined by the Lorentz potential leading to various interference patterns. Moreover, for some parameters, see Figure 11, we can observe extremely pronounced interference patterns inside the junction, such as whispering gallery modes [36, 69, 76. It is interesting to see that such modes can be induced by an external electron beam. 


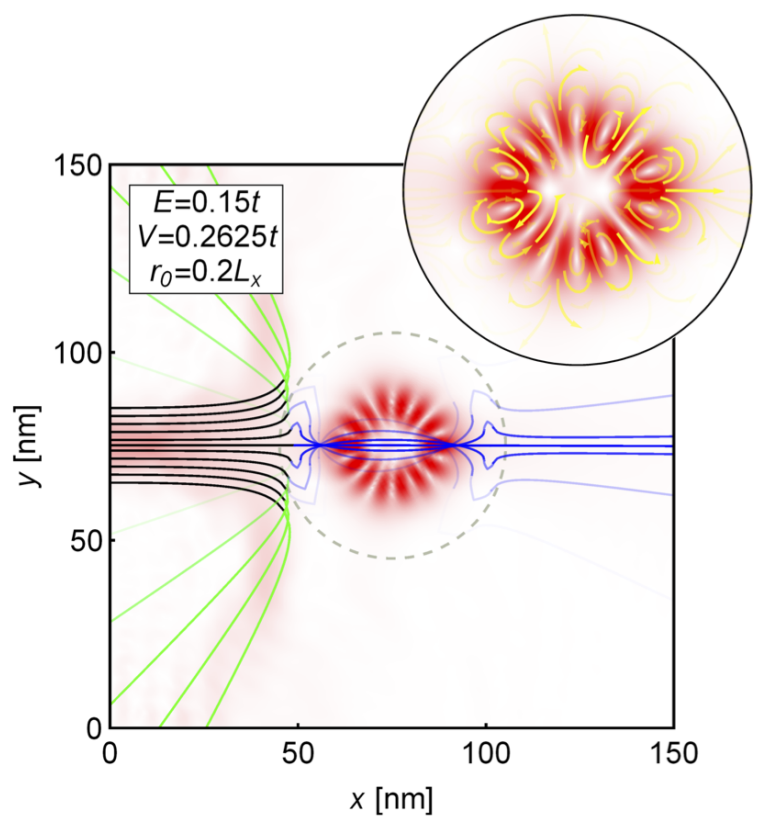

Figure 11. Gradient-index electron optics in smooth circular pn junctions. Strong interference patterns in the form of whispering gallery modes can be observed for specific parameters.

In Figure 12, we study the transition from smooth to abrupt circular pn junctions by increasing the parameter $\alpha$ in Equation (5). When the junction profile becomes more abrupt, the current density is dispersed more strongly and the focusing point of the current moves away from the center of the junction (see Figure 10 (b)) towards the left edge of the junction (see Figure 8 (b)). Moreover, interference patterns are observed inside the junction, which depend sensitively on the smoothness of the junction.

When studying smooth circular nn' and pp' junctions, see Figure 13, we observe that the current flow patterns change qualitatively and more drastically in comparison with straight junctions, see Figure 7. In the nn' regime, the Lorentz potential acts as a beam splitter, which separates even electrons with small angular momentum. The semi-classical trajectories indicate that the Klein tunneling persists for zero angular momentum (normal incidence). Once more, the trajectories from Equation (11) and numerical calculations of the current density show a remarkable agreement. Furthermore, a circular pp' junction behaves like a converging lens, see Figure 13 (b). The electron current flow is focused on a single point behind the junction. In contrast with the pn regime, interference patterns decrease, and therefore, pp' and nn' junctions are an ideal scenario to realize gradient-index electron optics. It is important to note that the refractive index defined by Equation (8) is fully positive in the nn' regime, while it is all negative in the pp' regime. Moreover, we have demonstrated that the gradient-index
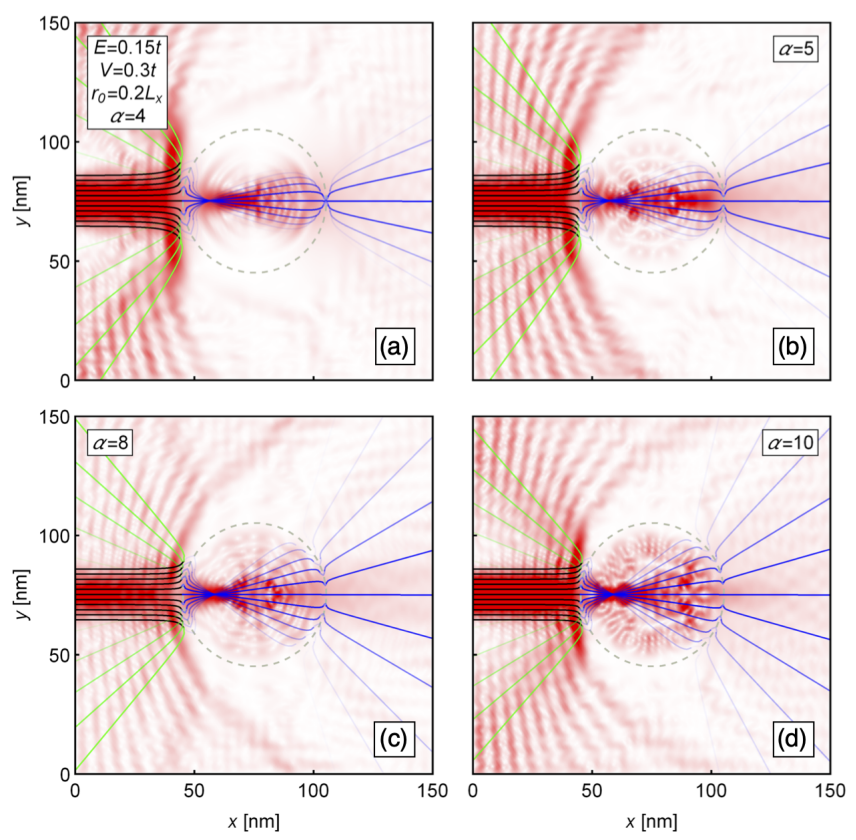

Figure 12. Current flow in circular pn junctions. When the parameter $\alpha$ is increased the junction changes more abruptly. The current density is dispersed more strongly and the focusing point moves from the center of the junction (see Figure 10 (b)) towards the left (see Figure 8 (b)). Moreover, distinct interference patterns can be observed inside the junction.
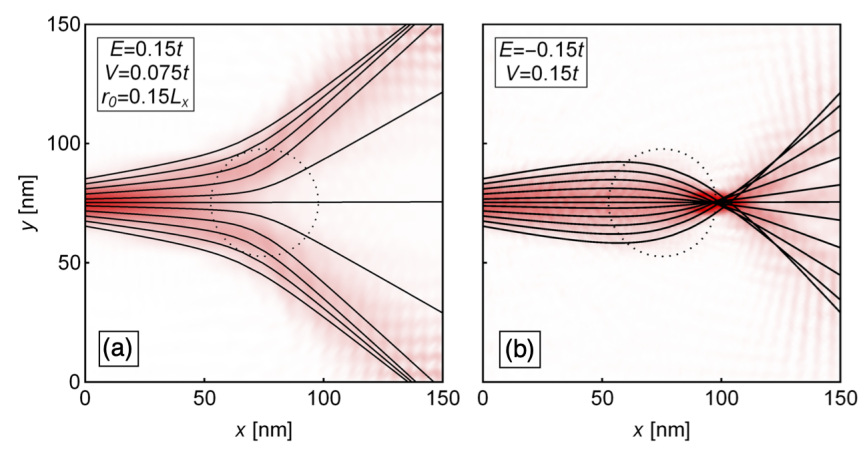

Figure 13. Current flow in smooth circular nn' (a) and pp' (b) junctions. The former represent efficient electron beam splitters, while the latter act as a converging lens.

electron optics is in line with the principles of light optics even for negative refraction, because "the rays bend towards the region of higher refractive index" [71]. Figure 14 shows the advantages of the optical methods used here over the relativistic geodesics. Apart of the classical regions where both approaches perfectly agree, we also see the tunneling through a forbidden region, corresponding to tracing the evanescent waves, and further propagation in a second classical region. In this way, we obtain a much more complete picture and better agreement with the wave dynamics.

As a proof of principle, we also apply the developed 


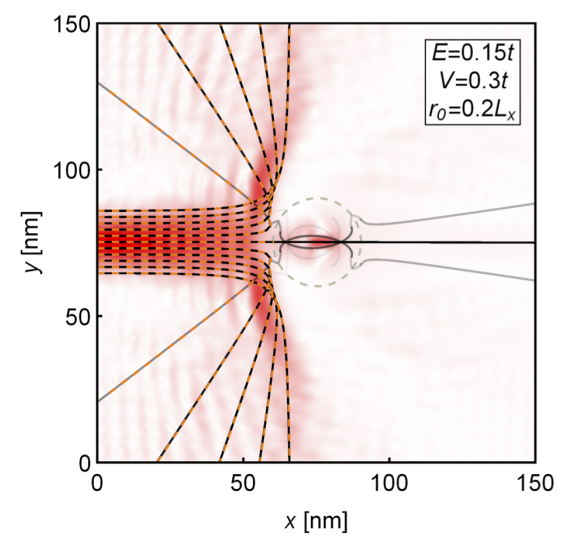

Figure 14. Current flow in a smooth circular pn junction. Semi-classical trajectories (see Equation (11)) are shown by solid-black curves, while the geodesics are given by the dashed-orange curves. Both approaches are equivalent for the reflected electrons. However, geodesics cannot be used to estimate the paths of the electrons that are transmitted through the junction.

techniques to the well known Luneburg and Maxwell gradient-index lenses. The standard Luneburg lens 77 is known for its perfect focusing of parallel beams coming from any direction and is described by

$$
n(\boldsymbol{r})=n_{0} \begin{cases}\sqrt{2-\left(r / r_{0}\right)^{2}} & \text { for } r<r_{0} \\ 1 & \text { otherwise. }\end{cases}
$$

In our situation, related to graphene, the interesting parameter is the potential $V(\boldsymbol{r})$ which follows from Equation (8) and $n_{0}=E / v_{F}$. The Maxwell's fish-eye lens [78] has all pairs of focusing points on a circle and is generated by the refractive index

$$
n(\boldsymbol{r})=\frac{n_{0}}{1+\left(r / r_{0}\right)^{2}},
$$

which is closely related to the previously used potential in Equation (5). The functionality of these electron optical devices in graphene in demonstrated in Figure 15.

\section{CONCLUSIONS}

In this paper, we investigated the ballistic current flow in smooth graphene pn junctions. Comparing numerically calculated current densities with semi-classical trajectories, we demonstrated that the current flow in these devices can be understood largely in terms of gradientindex optics. These semi-classical trajectories are an efficient tool to estimate the current flow paths in nanoelectronic devices.

In straight pn junctions, we confirmed the validity of a generalized Snell's law and reported additionally interference effects between the incident and reflected electron
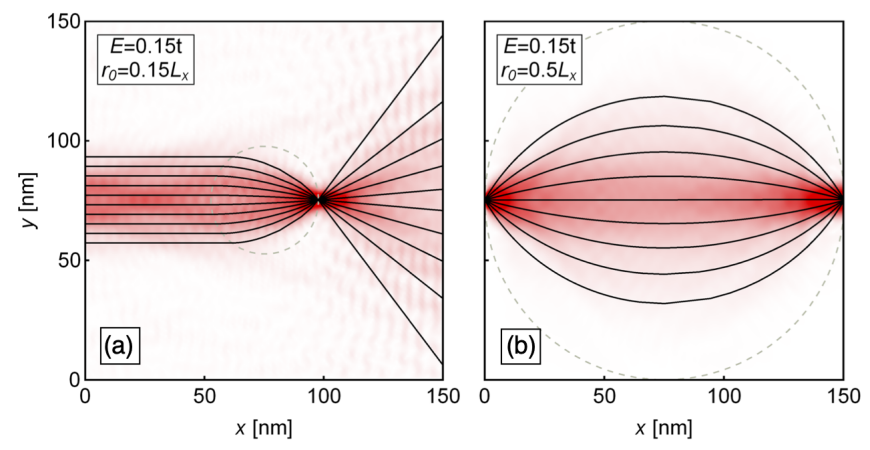

Figure 15. Current flow in graphene with an electrostatic potential that generates a Luneburg lens (a) and a Maxwell's fish-eye lens (b).

waves, see Figures 4- 6. Forbidden regions emerge in smooth junctions, see Figure 3. The current is reflected at the interface of these regions, except for the normally incident electrons due to Klein tunneling. Such smooth pn junctions can be used to generate narrow parallel electron beams.

In circular pn junctions with an abruptly changing profile, the part of the current density that enters the junction is focused onto a single point, which agrees with a caustic of the semi-classical trajectories, see Figure 8 . When the profile of the junction is smoothed, a circular junction with a gradually changing refractive index is obtained.The semi-classical trajectories agree qualitatively with the quantum current density but an energetically forbidden region intensifies the interference both outside and inside the junction, see Figure 10. This strong interference in smooth circular pn junction leads, for specific parameters, to interesting patterns such as whispering gallery modes, see Figure 11. Finally, we demonstrated in Figure 13 that smooth circular nn' and pp' junctions act as beam splitters and converging lenses, respectively. In Figure 15, we proved the feasibility of realizing Luneberg and Maxwell lenses in graphene.

We are confident that the presented broad variety of different properties of smooth graphene pn junctions will stimulate gradient-index optics experiments in graphene. Our findings contribute to the overall understanding of the local current flow in graphene and may eventually lead to new nano-electronic devices.

\section{ACKNOWLEDGMENTS}

EPR gratefully acknowledges a CONACYT graduate scholarship. EPR thanks N. Szpak and D. Wolf for their hospitality at the University Duisburg-Essen (Germany), where part of this research was performed. EPR, YBO and TS gratefully acknowledge funding from CONACYT Proyecto Fronteras 952, CONACYT Proyecto A1S-13469, and UNAM-PAPIIT IA103020. NS grate- 
fully acknowledges funding by the Deutsche Forschungsgemeinschaft (DFG, German Research Foundation) Project 278162697 - SFB 1242.

[1] G.-H. Lee, G.-H. Park, and H.-J. Lee, Nat. Phys. 11, 925 (2015).

[2] J. Cayssol, B. Huard, and D. Goldhaber-Gordon, Phys. Rev. B 79, 075428 (2009).

[3] J. R. Williams, T. Low, M. S. Lundstrom, and C. M. Marcus, Nat. Nanotechnol. 6, 222 (2011).

[4] B. Brun, N. Moreau, S. Somanchi, V.-H. Nguyen, K. Watanabe, T. Taniguchi, J.-C. Charlier, C. Stampfer, and B. Hackens, Phys. Rev. B 100, 041401 (2019).

[5] J. Cserti, A. Pályi, and C. Péterfalvi, Phys. Rev. Lett. 99, 246801 (2007).

[6] V. V. Cheianov, V. Fal'ko, and B. L. Altshuler, Science 315, 1252 (2007).

[7] J. L. Garcia-Pomar, A. Cortijo, and M. Nieto-Vesperinas, Phys. Rev. Lett. 100, 236801 (2008).

[8] P. E. Allain and J. N. Fuchs, Eur. Phys. J. B 83, 301 (2011).

[9] A. F. Young and P. Kim, Nat. Phys. 5, 222 (2009).

[10] S. Chen, Z. Han, M. M. Elahi, K. M. M. Habib, L. Wang, B. Wen, Y. Gao, T. Taniguchi, K. Watanabe, J. Hone, et al., Science 353, 1522 (2016).

[11] B. Huard, J. A. Sulpizio, N. Stander, K. Todd, B. Yang, and D. Goldhaber-Gordon, Phys. Rev. Lett. 98, 236803 (2007).

[12] A. H. C. Neto, F. Guinea, N. M. R. Peres, K. S. Novoselov, and A. K. Geim, Rev. Mod. Phys. 81, 109 (2009).

[13] K.-K. Bai, J.-J. Zhou, Y.-C. Wei, J.-B. Qiao, Y.-W. Liu, H.-W. Liu, H. Jiang, and L. He, Phys. Rev. B 97, 045413 (2018).

[14] X. Zhou, A. Kerelsky, M. M. Elahi, D. Wang, K. M. M. Habib, R. N. Sajjad, P. Agnihotri, J. U. Lee, A. W. Ghosh, F. M. Ross, et al., ACS Nano 13, 2558 (2019).

[15] J. Hu, A. F. Rigosi, J. U. Lee, H.-Y. Lee, Y. Yang, C.-I. Liu, R. E. Elmquist, and D. B. Newell, Phys. Rev. B 98, 045412 (2018).

[16] K.-K. Bai, J.-B. Qiao, H. Jiang, H. Liu, and L. He, Phys. Rev. B 95, 201406 (2017).

[17] M. I. Katsnelson, K. S. Novoselov, and A. K. Geim, Nat. Phys. 2, 620 (2006).

[18] V. V. Cheianov and V. I. Fal'ko, Phys. Rev. B 74, 041403 (2006).

[19] N. Stander, B. Huard, and D. Goldhaber-Gordon, Phys. Rev. Lett. 102, 026807 (2009).

[20] D. Schurig, J. J. Mock, B. J. Justice, S. A. Cummer, J. B. Pendry, A. F. Starr, and D. R. Smith, Science 314, 977 (2006).

[21] J. B. Pendry, Phys. Rev. Lett. 85, 3966 (2000).

[22] V. G. Veselago, Sov. Phys. Usp. 10, 509 (1968).

[23] N. Levy, S. A. Burke, K. L. Meaker, M. Panlasigui, A. Zettl, F. Guinea, A. H. C. Neto, and M. F. Crommie, Science 329, 544 (2010).

[24] F. Guinea, M. I. Katsnelson, and A. K. Geim, Nat. Phys. 6, $30(2010)$.

[25] T. Low and F. Guinea, Nano Lett. 10, 3551 (2010).
[26] T. Stegmann and N. Szpak, New J. Phys. 18, 053016 (2016).

[27] B. Amorim, A. Cortijo, F. de Juan, A. Grushin, F. Guinea, A. Gutiérrez-Rubio, H. Ochoa, V. Parente, R. Roldán, P. San-Jose, et al., Phys. Rep. 617, 1 (2016).

[28] G. G. Naumis, S. Barraza-Lopez, M. Oliva-Leyva, and H. Terrones, Rep. Prog. Phys. 80, 096501 (2017).

[29] R. L. Heinisch, F. X. Bronold, and H. Fehske, Phys. Rev. B 87, 155409 (2013).

[30] Y. Betancur-Ocampo, J. Phys.: Condens. Matter 30, 435302 (2018).

[31] Y. Betancur-Ocampo, Phys. Rev. B 98, 205421 (2018).

[32] W. Mu, G. Zhang, Y. Tang, W. Wang, and Z. Ou-Yang, J. Phys.: Condens. Matter 23, 495302 (2011).

[33] C. Péterfalvi, A. Pályi, and J. Cserti, Phys. Rev. B 80, 075416 (2009).

[34] S. Prabhakar, R. Nepal, R. Melnik, and A. A. Kovalev, Phys. Rev. B 99, 094111 (2019).

[35] N. A. (Garg), S. Ghosh, and M. Sharma, J. Phys.: Condens. Matter 26, 155301 (2014).

[36] Y. Jiang, J. Mao, D. Moldovan, M. R. Masir, G. Li, K. Watanabe, T. Taniguchi, F. M. Peeters, and E. Y. Andrei, Nat. Nanotechnol. 12, 1045 (2017).

[37] A. Mreńca-Kolasińska, S. Heun, and B. Szafran, Phys. Rev. B 93, 125411 (2016).

[38] M. A. Khan and M. N. Leuenberger, Phys. Rev. B 90, 075439 (2014).

[39] T. Stegmann and N. Szpak, 2D Mater. 6, 015024 (2018).

[40] F. Zhai, Y. Ma, and K. Chang, New J. Phys. 13, 083029 (2011).

[41] D. Zhai and N. Sandler, Phys. Rev. B 98, 165437 (2018).

[42] M. M. Grujić, M. Z. Tadić, and F. M. Peeters, Phys. Rev. Lett. 113, 046601 (2014).

[43] J. R. Schaibley, H. Yu, G. Clark, P. Rivera, J. S. Ross, K. L. Seyler, W. Yao, and X. Xu, Nat. Rev. Mat. 1, 16055 (2016).

[44] M. Settnes, J. H. Garcia, and S. Roche, 2D Mater. 4, 031006 (2017).

[45] M. Settnes, S. R. Power, M. Brandbyge, and A.-P. Jauho, Phys. Rev. Lett. 117, 276801 (2016).

[46] S. P. Milovanović and F. M. Peeters, Appl. Phys. Lett. 109, 203108 (2016).

[47] R. Carrillo-Bastos, M. Ochoa, S. A. Zavala, and F. Mireles, Phys. Rev. B 98, 165436 (2018).

[48] R. Carrillo-Bastos, C. León, D. Faria, A. Latgé, E. Y. Andrei, and N. Sandler, Phys. Rev. B 94, 125422 (2016).

[49] P. Rickhaus, P. Makk, K. Richter, and C. Schnenberger, Appl. Phys. Lett. 107, 251901 (2015).

[50] J. J. Wang, S. Liu, J. Wang, and J.-F. Liu, Phys. Rev. B 98, 195436 (2018).

[51] C.-H. Park, Y.-W. Son, L. Yang, M. L. Cohen, and S. G. Louie, Nano Lett. 8, 2920 (2008).

[52] M.-H. Liu, C. Gorini, and K. Richter, Phys. Rev. Lett. 118, 066801 (2017).

[53] R. N. Sajjad and A. W. Ghosh, Appl. Phys. Lett. 99, 123101 (2011).

[54] D. Gunlycke and C. T. White, Phys. Rev. B 90, 035452 (2014).

[55] H. Graef, Q. Wilmart, M. Rosticher, D. Mele, L. Banszerus, C. Stampfer, T. Taniguchi, K. Watanabe, J.-M. Berroir, E. Bocquillon, et al., Nat. Commun. 10, 2428 (2019).

[56] K. Wang, M. M. Elahi, L. Wang, K. M. M. Habib, T. Taniguchi, K. Watanabe, J. Hone, A. W. Ghosh, G.-H. 
Lee, and P. Kim, Proc. Nat. Acad. Sc. 116, 6575 (2019).

[57] Q. Wilmart, S. Berrada, D. Torrin, V. H. Nguyen, G. Fève, J.-M. Berroir, P. Dollfus, and B. Plaçais, 2D Mater. 1, 011006 (2014).

[58] P. Bøggild, J. M. Caridad, C. Stampfer, G. Calogero, N. R. Papior, and M. Brandbyge, Nat. Commun. 8, 15783 (2017).

[59] Y. Betancur-Ocampo, F. Leyvraz, and T. Stegmann, Nano Lett. 19, 7760 (2019).

[60] Y. Betancur-Ocampo, E. Paredes-Rocha, and T. Stegmann, arXiv:2006.12441.

[61] R. D. Y. Hills, A. Kusmartseva, and F. V. Kusmartsev, Phys. Rev. B 95, 214103 (2017).

[62] M. Yang, Q.-T. Hou, and R.-Q. Wang, New J. Phys. 21, 113057 (2019).

[63] M. Yang, Q.-T. Hou, and R.-Q. Wang, New J. Phys. 22, 033015 (2020).

[64] M. Lu and X.-X. Zhang, J. Phys.: Condens. Matter 30, 215303 (2018).

[65] S.-H. Zhang and W. Yang, New J. Phys. 21, 103052 (2019).

[66] L. Cohnitz, A. D. Martino, W. Husler, and R. Egger, Phys. Rev. B 94, 165443 (2016).

[67] J.-S. Wu and M. M. Fogler, Phys. Rev. B 90, 235402 (2014).

[68] B. Liao, M. Zebarjadi, K. Esfarjani, and G. Chen, Phys. Rev. B 88, 155432 (2013).
[69] Y. Zhao, J. Wyrick, F. D. Natterer, J. F. RodriguezNieva, C. Lewandowski, K. Watanabe, T. Taniguchi, L. S. Levitov, N. B. Zhitenev, and J. A. Stroscio, Science 348, 672 (2015).

[70] B. Brun, N. Moreau, S. Somanchi, V.-H. Nguyen, A. Mreńca-Kolasińska, K. Watanabe, T. Taniguchi, J.C. Charlier, C. Stampfer, and B. Hackens, 2D Mater. 7, 025037 (2020).

[71] M. Born and E. Wolf, Principles of Optics (Cambridge University Press, 1999).

[72] S. Datta, Electronic Transport in Mesoscopic Systems (Cambridge University Press, 1997).

[73] S. Datta, Quantum Transport: Atom to Transistor (Cambridge University Press, 2005).

[74] T. Low, S. Hong, J. Appenzeller, S. Datta, and M. S. Lundstrom, IEEE Trans. Electron Devices 56, 1292 (2009).

[75] R. N. Sajjad, S. Sutar, J. U. Lee, and A. W. Ghosh, Phys. Rev. B 86, 155412 (2012).

[76] F. Ghahari, D. Walkup, C. Gutiérrez, J. F. RodriguezNieva, Y. Zhao, J. Wyrick, F. D. Natterer, W. G. Cullen, K. Watanabe, T. Taniguchi, et al., Science 356, 845 (2017).

[77] R. K. Luneberg, Mathematical Theory of Optics (Brown University, Providence, 1944), pp. 189-213.

[78] J. Maxwell, The Cambridge and Dublin Mathematical Journal. 9, 911 (1854). 